\title{
Defect Effect of Bi-infinite Words in the Two-element Case
}

\author{
Ján Maňuch ${ }^{\dagger}$ \\ Department of Mathematics and Turku Centre for Computer Science, University of Turku, Finland \\ manuch@es.utu.fi
}

received Jul 18, 2000, accepted September, 2000.

Let $X$ be a two-element set of words over a finite alphabet. If a bi-infinite word possesses two $X$-factorizations which are not shiftequivalent, then the primitive roots of the words in $X$ are conjugates. Note, that this is a strict sharpening of a defect theorem for bi-infinite words stated in [KMP2].

Moreover, we prove that there is at most one bi-infinite word possessing two different $X$-factorizations and give a necessary and sufficient conditions on $X$ for the existence of such a word. Finally, we prove that the family of sets $X$ for which such a word exists is parameterizable.

Keywords: defect theorem, bi-infinite words

\section{Introduction}

Defect theorem is one of the fundamental results on words, cf [Lo]. Intuitively it states that if $n$ words satisfy a nontrivial relation, then these words can be expressed as products of at most $n-1$ words. Actually, as discussed in $[\mathrm{CK}]$, for example, there does not exist just one defect theorem but several ones depending on restrictions put on the required $n-1$ words.

It is also well-known that the nontrivial relation above can be replaced by a weaker condition, namely by the nontrivial one-way infinite relation, cf. $[\mathrm{Br}]$ and $[\mathrm{HK}]$. The goal of this note is to look for defect theorems for bi-infinite words. In a strict sense such results do not exist: the set $X=\{a b, b a\}$ of words satisfies a bi-infinite nontrivial relation since $(a b)^{\mathbb{Z}}=(b a)^{\mathbb{Z}}$, but there exists no word $\rho$ such that $X \subseteq \rho^{+}$. However, in [KMP2] there was proved one result and we are going to prove another one in a special case which both can be viewed as defect theorems for bi-infinite words.

In terms of factorizations of words defect theorem can be stated as follows: Let $X \subseteq \Sigma^{+}$be a finite set of words. If there exists a word $w \in \Sigma^{+}$having two different $X$-factorizations, then the rank of $X$ is at most $\operatorname{card}(X)-1$. Here the rank of $X$ can be defined in different ways, cf again [CK]. For example, it can be defined as a combinatorial rank $r_{\mathrm{c}}(X)$ denoting the smallest number $k$ such that $X \subseteq Y^{+}$with $\operatorname{card}(Y)=k$.

\footnotetext{
${ }^{\dagger}$ Supported by Academy of Finland under Grant No. 14047.

1365-8050 @ 2001 Maison de l'Informatique et des Mathématiques Discrètes (MIMD), Paris, France
} 
To describe our results let $w$ be a bi-infinite word, i.e., an element of $\Sigma^{\mathbb{Z}}$, and $X$ a finite subset of $\Sigma^{+}$. We say that $w$ has an $X$-factorization if $w \in X^{\mathbb{Z}}$, and that $w$ has two different $X$-factorizations, if it has two $X$-factorizations such that they do not match at least in one point of $w$. The following result was shown in [KMP2]:

If a nonperiodic bi-infinite word $w$ has two different $X$-factorizations, then the combinatorial rank $r_{\mathrm{c}}(X)$ of $X$ is at most $\operatorname{card}(X)-1$. Moreover, if $r_{\mathrm{c}}(X)=\operatorname{card}(X)$, then the number of bi-infinite words with two different $X$-factorizations is finite.

We are going to prove a strict sharpening of this result for the two-element case:

Let $\operatorname{card}(X)=r_{\mathrm{c}}(X)=2$, so that $X$ is a code. If a bi-infinite word $w$ has two different $X$-factorizations which are not shiftequivalent, then the primitive roots of words in $X$ are conjugates. Moreover, there is at most one bi-infinite word possessing two different $X$-factorizations.

The first part of our result is related to the main result of [IRIR], and, we believe, deducible from considerations of that paper. However, our proof is self-contained and essentially shorter, and moreover formulated directly to yield a defect-type of theorem.

Our paper is organized as follows.

In Section 2 we fix our terminology and present the auxiliary results needed for our proofs. In Section 3 we prove, as our main result, a defect theorem for binary sets $X$ satisfying a nontrivial bi-infinite relation. To prove this seems to be quite complicated. In Section 4 we prove the second part of our result, i.e., the uniqueness of the $X$-ambiguous bi-infinite word in the two-element case. In Section 5 we give a characterization of two-element sets $X$, which allow an $X$-ambiguous bi-infinite word. The last section contains conclusions and open problems.

The extended abstract of this paper and paper [KMP2] has appeared in [KMP1].

\section{Preliminaries}

In this section we fix our terminology and recall a few lemmas on combinatorics of words needed for the proofs of our results. For undefined notions we refer to [Lo] or [CK].

Let $\Sigma$ be a finite alphabet and $X$ a finite subset of $\Sigma^{+}$. The sets of all finite, infinite and bi-infinite words over $\Sigma$ are denoted by $\Sigma^{*}, \Sigma^{\mathbb{N}}$ and $\Sigma^{\mathbb{Z}}$, respectively. Formally, a representation of bi-infinite word is a mapping $f_{w}: \mathbb{Z} \rightarrow \Sigma$, usually written as

$$
w=\ldots a_{-1} a_{0} a_{1} \ldots \quad \text { with } a_{i}=f_{w}(i) .
$$

Representations $f: \mathbb{Z} \rightarrow \Sigma$ and $f^{\prime}: \mathbb{Z} \rightarrow \Sigma$ represent the same bi-infinite word if there exists an integer $i_{0}$ such that for all integers $i, f(i)=f^{\prime}\left(i_{0}+i\right)$.

Let $f_{w}$ be a representation of a bi-infinite word $w$. We say that a bi-infinite word is periodic if there exists a positive integer $i_{0}$, called $a$ period, such that $f_{w}(i)=f_{w}\left(i_{0}+i\right)$ for all integers $i$. Note that a non-periodic bi-infinite word has infinitely many representations, while a periodic one has exactly $\pi(w)$ representations, where $\pi(w)$ is the smallest period of $w$. 
An $X$-factorization of $w$ is any sequence of words from $X$ yielding $w$ as their products. Formally, let $f_{w}$ be a fixed representation of $w \in \Sigma^{\mathbb{Z}}$. An $X$-factorization of $w$ is a mapping $F: \mathbb{Z} \rightarrow X \times \mathbb{Z}$ such that for each $k \in \mathbb{Z}$ if $F(k)=(\alpha, i)$ and $F(k+1)=(\beta, j)$, then $a_{i} a_{i+1} \ldots a_{j-1}=\alpha$, i.e., the position $i$ is a starting position of the factor $\alpha$ in $w$. We say that two $X$-factorizations $F_{1}$ and $F_{2}$ of a bi-infinite word are

- different, whenever there is a $k_{0} \in \mathbb{Z}$ such that for each $k \in \mathbb{Z}, F_{1}\left(k_{0}\right) \neq F_{2}(k)$,

- disjoint, whenever the starting positions of all factors in $F_{1}$ are distinct from the ones in $F_{2}$,

- shiftequivalent, if there is a $k_{0}$ such that whenever $F_{1}(k)=(\alpha, i)$ and $F_{2}\left(k_{0}+k\right)=(\beta, j)$, then $\alpha=\beta$.

Notice that the above definitions are independent on the choice of a representation of $w$.

An $X$-ambiguous bi-infinite word is a bi-infinite word, which has two different $X$-factorizations. Let $\operatorname{amb}(X)$ be the set of all $X$-ambiguous bi-infinite words and let $\operatorname{sum}(X)$ be the sum of lengths of words in $X$, i.e., the size of $X$.

Example 1. Let $X=\{a, b a b, b a a b\}$. The word $(b a a)^{\mathbb{Z}}$ has two different $X$-factorizations, namely the ones depicted as:

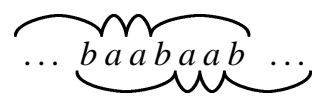

They are clearly shiftequivalent. On the other hand the word

$$
w=\ldots \text { bababaabaab } \cdots={ }^{\mathbb{N}}(b a) b(a a b)^{\mathbb{N}}
$$

also has two different $X$-factorizations, which, however, are not shiftequivalent:

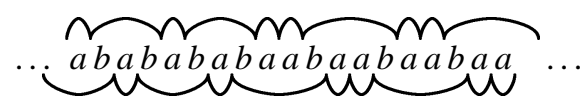

Clearly, in both of the above cases the two factorizations are disjoint.

We define the combinatorial rank of $X \subseteq \Sigma^{+}$by the formula

$$
r_{\mathrm{c}}(X)=\min \left\{\operatorname{card}(Y) \mid X \subseteq Y^{+}\right\}
$$

For the sake of completeness we remind that

$$
r_{\mathrm{c}}(X) \leq r_{\mathrm{f}}(X) \leq \operatorname{card}(X)
$$

where $r_{\mathrm{f}}(X)$ denotes the free rank (or simply the rank) of $X$ defined as the cardinality of the base of the smallest free semigroup containing $X, \mathrm{cf}[\mathrm{CK}]$.

Example 1 (continued). Clearly, $r_{\mathrm{c}}(X)=2$, since $X \subseteq\{a, b\}^{+}$, but for no word $\rho$ the inclusion $X \subseteq \rho^{+}$ holds. On the other hand, since $X$ is a code we conclude that $r_{\mathrm{f}}(X)=3$. 
We say that a finite word $w=w_{1} \ldots w_{m}$ has a period $n \in \mathbb{N}$, if there is a word $u$ such that $w=u^{n}$. The shortest period is called the period of $w$, denoted as $\pi(w)$. If $w=u^{\pi(w)}$, then $u$ is called the root of $w$, denoted as $\rho(w)$. A word $w$ is primitive if $\rho(w)=w$. The mirror image of $w$, denoted $w^{\mathrm{R}}$, is the word $w_{m} \ldots w_{1}$.

Next we recall a few basic results on words that we shall need in our later considerations, for their proofs the reader is referred to [Lo] or [CK].

Lemma 1. ( $\mathrm{Fine}$ and $\mathrm{Wilf}$ ) Let $u, v \in \Sigma^{+}$. If the words $u^{\mathbb{N}}$ and $v^{\mathbb{N}}$ have a common prefix of length at least $|u|+|v|-\operatorname{gcd}(|u|,|v|)$, then $u$ and $v$ are powers of a common word.

Lemma 2. No primitive word $r$ satisfies a relation $r r=\operatorname{srp}$ with $s \neq 1$ and $p \neq 1$.

Lemma 3. If two words $u$ and $v$ satisfy the relation $u t=t v$ for some $u, v, t \in \Sigma^{+}$, i.e., if they are conjugates, then there exist words $p$ and $q$ such that $p q$ is primitive and

$$
u=(p q)^{i}, \quad v=(q p)^{i} \quad \text { and } \quad t \in p(q p)^{*} \quad \text { for some } i \geq 1
$$

In Section 4 we shall need also the following result which has been proved in [LyS].

Lemma 4. Consider nonempty words $x, y, z$ satisfying equation $x^{m}=y^{n} z^{p}$, where $m, n, p \geq 2$. Then all words $x, y, z$ are powers of a common word.

In order to formulate our fifth, and most crucial lemma, we need some terminology, cf [CK] or [HK]. We associate a finite set $X \subseteq \Sigma^{+}$with a graph $\mathcal{G}_{X}=\left(V_{X}, E_{X}\right)$, called the dependency graph of $X$, as follows: the set $V_{X}$ of vertices of $\mathcal{G}_{X}$ equals to $X$, and the set $E_{X}$ of edges of $\mathcal{G}_{X}$ is defined by the condition

$$
(x, y) \in E_{X} \quad \text { iff } \quad x X^{\mathbb{N}} \cap y X^{\mathbb{N}} \neq \emptyset .
$$

Then we have

Lemma 5. For each finite set $X \subseteq \Sigma^{+}$, the combinatorial rank of $X$ is at most the number of connected components of $\mathcal{G}_{X}$.

As we shall see, Lemma 5 is particularly suitable for our subsequent considerations. Indeed, in that lemma it is crucial that words in $X$ are nonempty, and that indeed is satisfied in the proofs of our Theorem 2 .

\section{The Two-element Case}

In this section we generalize the following result of [KMP2] in the case of two-element sets.

Theorem 1. Consider a set $X=\left\{\alpha_{1}, \ldots, \alpha_{n}\right\} \subseteq \Sigma^{+}$. Let $w$ be a bi-infinite word over $\Sigma$ and $F_{1}, F_{2}$ two different $X$-factorizations of $w$. Then the combinatorial rank of $X$ is at most $n-1$, or both the word $w$ and the $X$-factorizations $F_{1}, F_{2}$ are periodic. Moreover, if the rank of $X$ is $n$, then the number of periodic bi-infinite words with two different $X$-factorizations is finite.

A restriction of Theorem 1 to two-element sets yields the following consequence.

Corollary 1. Consider set $X=\{\alpha, \beta\} \subseteq \Sigma^{+}$. Let $w$ be a bi-infinite word over $\Sigma$ and $F_{1}, F_{2}$ two different $X$-factorizations of $w$. Then the words $\alpha, \beta$ commute or both the word $w$ and the $X$-factorizations $F_{1}, F_{2}$ are periodic. 
First we recall that in a strict sense we cannot have a defect theorem for bi-infinite words even in this simple case.

Example 2. The set $X=\{a b, b a\}$ is of combinatorial rank 2 although the word $(a b)^{\mathbb{Z}}$ has two disjoint, and even non-shiftequivalent, $X$-factorizations.

As a main result of this paper we, however, show that the above example, and its natural variants, are the only exceptions which may occur. And even in these cases the roots of words in $X$ are conjugates, i.e., they are cyclic permutations of powers of a common word.

To prove our main result we will need also one partial result from [KMP2], which can be stated as follows:

Lemma 6. Let $X \subseteq \Sigma^{+}$and let

$$
w=\ldots w_{-2} w_{-1} w_{0} w_{1} w_{2} \ldots
$$

be a bi-infinite word. If there exists words $f_{1}, f_{2}, f_{1}^{\prime}, f_{2}^{\prime} \in X^{+}$, a word $t \in \Sigma^{+}$and integers $i<j<k<l$, $i^{\prime}<j^{\prime}<k^{\prime}<l^{\prime}$, such that

$$
\begin{array}{cc}
t=w_{i} \ldots w_{j-1}=w_{k} \ldots w_{l-1}=w_{i^{\prime}} \ldots w_{j^{\prime}-1}=w_{k^{\prime}} \ldots w_{l^{\prime}-1}, \\
f_{1}=w_{j} \ldots w_{l-1}, & f_{1}^{\prime}=w_{j^{\prime}} \ldots w_{l^{\prime}-1}, \\
f_{2}=w_{i} \ldots w_{k-1}, & f_{2}^{\prime}=w_{i^{\prime}} \ldots w_{k^{\prime}-1},
\end{array}
$$

then either $f_{1}$ and $f_{1}^{\prime}$ (resp. $f_{2}$ and $f_{2}^{\prime}$ ) commute, or $r_{c}(X)<\operatorname{card}(X)$.

In the notation of [KMP2] this lemma claims that the situation when $w$ possesses two different minimal $t$-pairs implies a defect effect. This situation is depicted in Figure 1 .

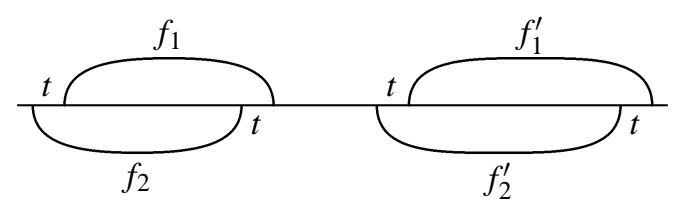

Fig. 1. An illustration of the situation considered in Lemma 6 .

Theorem 2. Consider set $X=\{\alpha, \beta\}$ with $\alpha, \beta \in \Sigma^{+}$. Let $w$ be a bi-infinite word over $\Sigma$ and $F_{1}, F_{2}$ two different $X$-factorizations of $w$ containing together both elements of $X$. Then one of the following possibilities holds:

(i) $\alpha$ and $\beta$ commute, or

(ii) the roots of $\alpha$ and $\beta$ are conjugates and $F_{1} \in \alpha^{\mathbb{Z}}, F_{2} \in \beta^{\mathbb{Z}}$, or vice versa, or

(iii) the two $X$-factorizations $F_{1}, F_{2}$ are shiftequivalent and there exists an $n \geq 1$ such that $F_{1}, F_{2} \in$ $\left(\alpha \beta^{n}\right)^{\mathbb{Z}}$ and $\alpha$ is primitive or $F_{1}, F_{2} \in\left(\beta \alpha^{n}\right)^{\mathbb{Z}}$ and $\beta$ is primitive.

Proof. We can assume that $\alpha$ and $\beta$ do not commute.

Then, by Lemma 5, the factorizations $F_{1}, F_{2}$ must be disjoint. Indeed, if factorizations $F_{1}$ and $F_{2}$ are not disjoint, then we can take the parts of factorizations to the right (respectively, to the left) from a 
place where they are joint to obtain an infinite equation $x_{1} x_{2} \cdots=y_{1} y_{2} \ldots$ over $X$ (respectively, $x_{1}^{\mathrm{R}} x_{2}^{\mathrm{R}} \cdots=$ $y_{1}^{\mathrm{R}} y_{2}^{\mathrm{R}} \ldots$ over $\left.X^{\mathrm{R}}\right)$. Since the factorizations are different, at least one of these two equations is nontrivial. Hence, by Lemma 5 , the words $\alpha$ and $\beta$ commute, a contradiction.

Further, by Corollary [1, the factorizations $F_{1}, F_{2}$ are periodic. By Lemma 1 the periods of $F_{1}$ and $F_{2}$ have the same length and are conjugates. Whenever we find the situation which is shown in Figure \&, since the factorizations are periodic with the same length of the periods, this situation occurs infinitely many times. Using Lemma 6 we get that $f_{1}, f_{2}$ are periods of $F_{1}, F_{2}$.

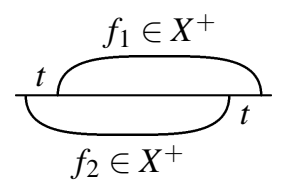

Fig. 2. In the situation depicted in the picture $f_{1}\left(f_{2}\right)$ is a part of the factorization $F_{1}$ (of the factorization $F_{2}$ ).

If both $\alpha$ and $\beta$ are not primitive, we can replace them by powers of their roots $\rho(\alpha)^{\pi(\alpha)}, \rho(\beta)^{\pi(\beta)}$ and explore the situation over a slightly different set $X=\{\rho(\alpha), \rho(\beta)\}$. If we prove that the claim holds for $\rho(\alpha), \rho(\beta)$, then, as is obvious, it must hold also for $\alpha$ and $\beta$ and, moreover, in case (iii) we have either $\pi(\alpha)=1$, i.e., $\alpha$ is primitive, or $\pi(\beta)=1$, i.e., $\beta$ is primitive. So it is enough to consider only the case when $\alpha$ and $\beta$ are primitive.

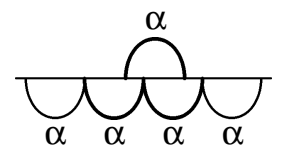

Fig. 3. An illustration of the situation when $F_{2}=\alpha \mathbb{Z}$ and $F_{1}$ contains $\alpha$.

Without loss of generality we can also assume that $|\alpha| \leq|\beta|$. Now, if $F_{2}$ does not contain the factor $\alpha \beta$, then it contains only $\alpha$ 's or only $\beta$ 's or there is a point inside $F_{2}$ from which to the left there are only $\beta$ 's and to the right only $\alpha$ 's. In the last case the factorization $F_{2}$ is clearly nonperiodic - a contradiction with Corollary 11. Consider now, for example, the case $F_{2}=\alpha \mathbb{Z}$. If $F_{1}$ contains any $\alpha$, then we have the situation depicted in Figure 3 which, by Lemma 2 , contradicts the primitiveness of $\alpha$. So we have $F_{1}=\beta^{\mathbb{Z}}$, and, by Lemma 1 , $\alpha$ and $\beta$ must be conjugates, which is case (ii).
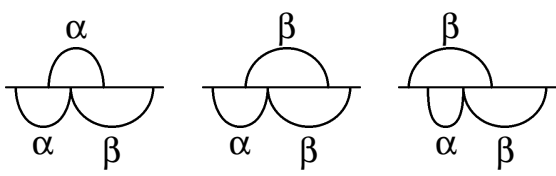

Fig. 4. All possible coverings of factor $\alpha \beta$ in $F_{2}$.

From now on we may assume that $F_{2}$ contains the factor $\alpha \beta$. In Figure 4 we can see all possibilities how $F_{1}$ covers the border between the above occurrences of $\alpha$ and $\beta$. We shall analyze all three cases. 


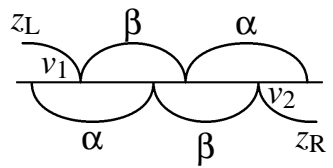

Fig. 5. The second case of 3 cases shown in Figure 4 .

Case 1,2. We can analyze first two cases simultaneously, because when we forget about the relation between lengths of $\alpha$ and $\beta$, then they are clearly symmetric. So consider the second case of 3 possibilities drawn in Figure 4 . If the word to the right of the $\beta$ in the factorization $F_{1}$ is also $\beta$, then $\beta$ is not primitive which is not the case. Hence, we have the situation shown in Figure 5. Now if $z_{\mathrm{R}}=\alpha$ or $z_{\mathrm{L}}=\alpha$, then $v_{1}=v_{2}$ and we arrive into the situation depicted in Figure 2 with $f_{1}=\beta \alpha$ and $f_{2}=\alpha \beta$ which is case (iii) of our claim. So consider the other case when $z_{\mathrm{R}}=z_{\mathrm{L}}=\beta$. We can continue in this way inductively until sequences of $\beta$ 's exceed $\alpha$ 's (on both sides at the same time) or we obtain the situation in Figure 2 with $f_{1}=\beta^{n} \alpha, f_{2}=\alpha \beta^{n}$, for some $n \geq 1$, which is again case (iii). The first possibility is shown in Figure 6 .

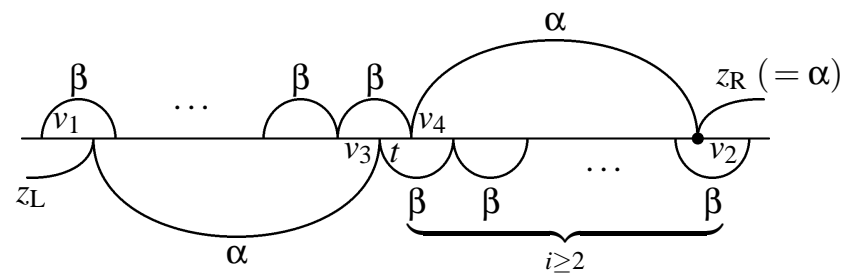

Fig. 6. The situation when sequences of $\beta$ 's exceed $\alpha$ 's on both sides.

Now again if $z_{\mathrm{R}}=\beta$, then we have $v_{1}=v_{2}$, and hence we are again in case (iii). So assume that $z_{\mathrm{R}}=\alpha$. We have $\beta=v_{3} t=t v_{4}$, which by Lemma 3 allows us to write $v_{3}=(p q)^{k}, v_{4}=(q p)^{k}, t=p(q p)^{n}$, where $p q$ is primitive and $k \geq 1, n \geq 0$. We can see that $\alpha$ ends with $p q$ and starts with $q p$. This means that the word $p q q p$ matches the word $\beta=(p q)^{k+n} p$ around the black point shown in Figure 6. Since the factorizations are disjoint, the black point must lie inside $\beta$. There are 5 possibilities where the black point inside $\beta$ can be. In case (1) the black point matches with the end of the first $p$ in $\beta$, in case (2) it matches with the end of any $p q$ in $\beta$, in case (3) it occurs inside the first $p$ of $\beta$, in case (4) inside the first $q$, and, finally, in the last case it occurs in the rest of $\beta$, as it is shown in Figure 7 .

(1) (2) $\ldots$

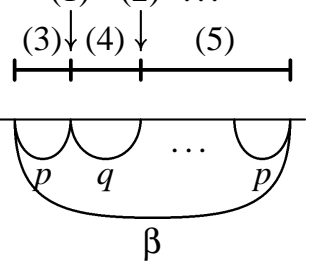

Fig. 7. 3 possibilities where the black point can occur in $\beta$.

In case (1) we have, according to Figure 6, the following two equations with unknowns $Y=\{\alpha, p, q\}$ :

$$
\alpha=v_{4} \beta^{i-2} p=q w_{1}, \quad \alpha=p \beta^{i-2} v_{3}=p w_{2}, \quad \text { where } w_{1}, w_{2} \in Y^{*} .
$$


The dependency graph of this system is then connected which implies that unknowns in $Y$, and hence also $\alpha$ and $\beta$, commute, which is a contradiction.

Similarly, in case (2) we can write

$$
\alpha=v_{4} \beta^{i-2}(p q)^{l}, \alpha e_{1} \beta^{i} \alpha=p(q p)^{k+n-l} e_{2} \alpha \beta^{i-1}(p q)^{l},
$$

where $l \geq 1, e_{1}, e_{2} \in X^{*}$ and the second equation is obtained by taking parts of $F_{1}, F_{2}$ between the black point and the next occurrence (to the right) of the black point, so $e_{i}$ is a part of the factorization $F_{i}$. We can rewrite these two equations as a system of equations with unknowns $Y=\{\alpha, p, q\}$ :

$$
\alpha=q p w_{1}, \quad \alpha w_{2}=p w_{3}, \quad \text { where } w_{1} \in\{p, q\}^{*}, w_{2}, w_{3} \in Y^{*},
$$

and, by Lemma 5 , we have again a contradiction.

In case (3) the $q p$, which follows the black point, lies inside the first $p q p$ in $\beta$. But this is a contradiction because then $p q$ cannot be primitive. In case (5) we can use the same argument with the $p q$ which precedes the black point. The situation around the black point in case (4) is shown in Figure 8.

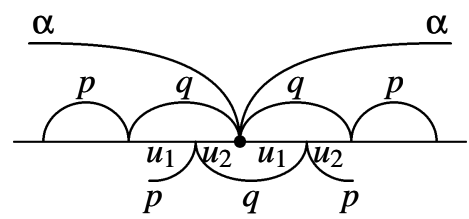

Fig. 8. The situation around the black point in case (4).

It follows, by Lemma 2, that $q$ is not primitive, and that there is an $s$ such that $u_{1}=s^{i}, u_{2}=s^{j}$ and $q=s^{i+j}$ with $i, j \geq 1$. Now, as above, we have two equations with unknowns $Y=\{\alpha, p, s\}$ :

$$
\begin{gathered}
\alpha=v_{4} \beta^{i-2} p u_{2}=s^{i+j} w_{1}, \\
s^{j} w_{1} e_{1} \alpha=u_{1}^{-1} \alpha e_{1} \alpha=(p q)^{k+n-1} p e_{2} \alpha \beta^{i-1} p u_{2}=p w_{2},
\end{gathered}
$$

where $w_{1}, w_{2} \in Y^{*}$ and $e_{1}, e_{2} \in X^{*}$ are parts of the $X$-factorizations. Note that the second equation deals with the word starting with the first $u_{2}$ after the black point and ending in the next occurrence of the black point.

Case 3. Now we shall analyze the third possibility shown in Figure 4 . Since $\beta$ is primitive there must be $\alpha$ to the right of the $\beta$ on the upper line. Using the same considerations as in the previous case we come to Figure 9, or we end up in case (iii) with $f_{1}=\beta \alpha^{n}, f_{2}=\alpha^{n} \beta$ for some $n \geq 1$. In the first case we have $\alpha=v_{1} v_{3}=v_{4} v_{2}$, where $\left|v_{1}\right|=\left|v_{2}\right|$ and $\left|v_{3}\right|=\left|v_{4}\right|$. There are again two possibilities.

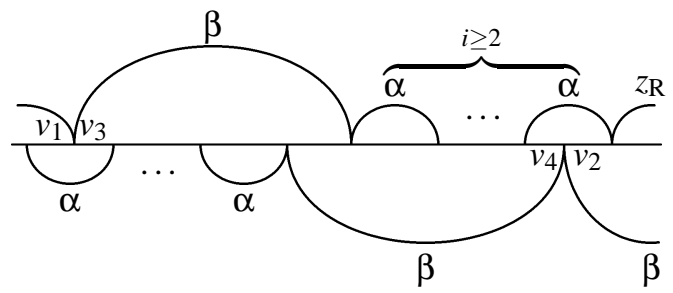

Fig. 9. The situation in Case 3. 
Assume first that $z_{\mathrm{R}}=\alpha$. We know that $\beta$ starts with $v_{3} v_{1}$, so, as it is shown in Figure 10, $v_{3} v_{1}$ lies inside $\alpha \alpha=v_{1} v_{3} v_{1} v_{3}$. This implies that either $\alpha$ is not primitive, or $v_{3} v_{1}$ matches with $v_{3} v_{1}$ in $\alpha \alpha$. In the first case we have a contradiction. In the second case it is obvious from Figure 10 that $v_{2}=v_{3}$, say equal to $p$, and $v_{1}=v_{4}$, say equal to $q$, and moreover that $|p|=\left|v_{3}\right|=\left|v_{4}\right|=|q|$. So we have $p \alpha^{i-1} \beta=\beta \alpha^{i-1} q$, which means that $v=p \alpha^{i-1}=p(q p)^{i-1}$ conjugates with $u=\alpha^{i-1} q=q(p q)^{i-1}$. We shall show that this is again a contradiction with the primitiveness of $\alpha=q p$.

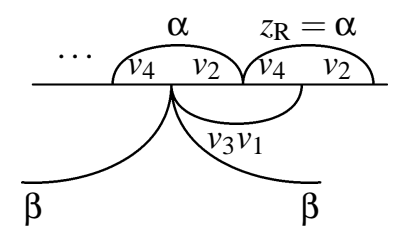

Fig. 10. The case $z_{\mathrm{R}}=\alpha$.

We have already analyzed this situation. Since the word $u$ is a conjugate with $v$, a factor of the word $u u$ must be equal to the word $v$. The word $u$ starts with $q p$ and ends with $p q$, so the middle point of the word $p q q p$ lies inside the word $v=p(q p)^{i-1}$. There are again 5 possibilities (see Figure 7). Since $|p|=|q|$ in cases (1) and (2) we have $p=q$, so that $\alpha=v_{1} v_{3}=q p=p^{2}$ proving that $\alpha$ is not primitive, a contradiction. In cases (3) and (5) we also have a contradiction with the primitiveness of $\alpha$ as we already proved. In case (4) we have $u_{1}=s^{i}, u_{2}=s^{j}, q=s^{i+j}$ (see Figure 8), and since $|p|=|q|$ we also have $p=u_{2} u_{1}=s^{i+j}=q$, which is again a contradiction.

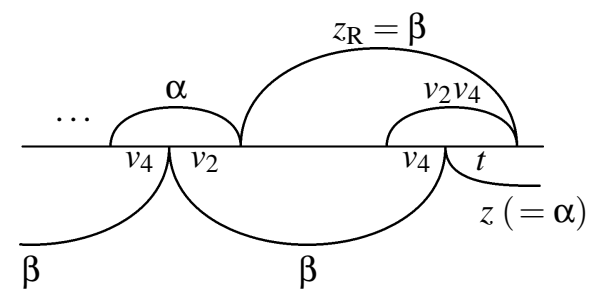

Fig. 11. The case $z_{\mathrm{R}}=\beta$.

So it remains the case $z_{\mathrm{R}}=\beta$. The situation is drawn in Figure 11. Since $\beta$ is not primitive $z$ must be $\alpha$. It is obvious that $|t|=\left|v_{2}\right|=\left|v_{1}\right|$, which implies $t=v_{1}$. We know that $\beta$ ends with $v_{2} v_{4}$, and hence there is $v_{2} v_{4}$ at the end of the last upper $\beta$ in Figure [11, and $v_{4}$ at the end of the last lower $\beta$. But since $\left|v_{2} v_{4}\right|=\left|v_{4} t\right|$ we have the equation $v_{2} v_{4}=v_{4} t=v_{4} v_{1}$. According to Figure 9 and Figure 11 we have the following system of equations with unknowns $Y=\left\{\beta, v_{1}, v_{2}, v_{3}, v_{4}\right\}$ :

$$
\begin{aligned}
v_{2} v_{4} & =v_{4} v_{1}, & v_{3}\left(v_{1} v_{3}\right)^{i-1} \beta & =\beta\left(v_{4} v_{2}\right)^{i-1} v_{4}, \\
(\alpha=) v_{1} v_{3} & =v_{4} v_{2}, & v_{2} \beta & =\beta v_{1} .
\end{aligned}
$$

The dependency graph associated with this system is connected, and hence all unknowns commute, in particular $\alpha$ commutes with $\beta$. This completes the proof of the theorem. 
Theorem $\unrhd$ deserves a few comments. The number of different $X$-factorizations of the bi-infinite word $w$ having an $X$-factorization is very different in cases ( $i$ - - (iii). In case ( $i$ ) there exist non-denumerably many such $X$-factorizations, in case $(i i)$ there are finitely many different $X$-factorizations, and if we consider all shiftequivalent $X$-factorizations as the one, then there are exactly two of them. Finally, in case (iii) there are also finitely many different $X$-factorizations, which are all shiftequivalent. This actually means that in case (iii) no bi-infinite word can be expressed in two different ways as a product of words from $X$. Hence, indeed, Theorem 2 shows a defect effect of a two-element set for bi-infinite factorizations.

In Theorem 2 we showed that if the words of $X$ do not commute and their roots are not conjugates, then only the case (iii) is possible. But if they do not commute and are conjugates Theorem $Z$ allows either case (ii) or ( $\mathrm{iii}$ ). Now we shall prove that in this situation only case (ii) is possible. According to the last part of the proof of Theorem \&, we can formulate the following lemma.

Lemma 7. If $p q$ is primitive and $p, q$ are nonempty, then $p(q p)^{n}$ and $q(p q)^{n}$ are not conjugates for any $n \geq 1$.

This yields easily

Corollary 2. If $\alpha$ and $\beta$ are different conjugates, then $\alpha \beta$ must be primitive.

Proof. Assume the contrary that $\alpha \beta$ is not primitive, so we have $\alpha \beta=t^{i}$, where $t$ is primitive and $i \geq 2$. Now if $i$ is even, then immediately $\alpha=\beta$, which is a contradiction. For odd $i=2 n+1$ we have $\alpha=t^{n} p$, $\beta=q t^{n}$, where $t=p q$. But $\alpha$ and $\beta$ are conjugates and so, by Lemma 7 , we have a contradiction.

In fact Corollary 2 is a special case of the claim in [LeS] which states under the additional assumption that $\alpha, \beta$ are primitive, that $\alpha \beta^{m}$ is primitive for all natural numbers $m$. The proof is not difficult, but we need only this special case to prove the next result.

Corollary 3. Consider set $X=\{\alpha, \beta\}$ with $\alpha, \beta \in \Sigma^{+}$. Let $w$ be a bi-infinite word over $\Sigma$ and $F_{1}, F_{2}$ two different $X$-factorizations of $w$ containing together both elements of $X$. If the roots of $\alpha, \beta$ are noncommuting conjugates, then $F_{1} \in \alpha^{\mathbb{Z}}, F_{2} \in \beta^{\mathbb{Z}}$, or vice versa.

Proof. Again as in the proof of Theorem §, we can assume that $\alpha, \beta$ are primitive. We have to show that the one of the $X$-factorizations $F_{1}, F_{2}$ consists only of $\alpha$ 's and the other only of $\beta^{\prime} s$. So assume the contrary that the lower factorization contains both $\alpha$ and $\beta$. Without loss of generality we have the situation shown in Figure [12.

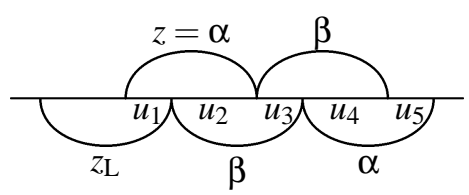

Fig. 12. The situation when $\alpha$ and $\beta$ are conjugates and the lower factorization contains both $\alpha$ and $\beta$.

Since $\beta$ is primitive we have $z=\alpha$, by Lemma 2. We can write $\beta=u_{2} u_{3}=u_{3} u_{4}$, and so, by Lemma 3 , we have

$$
u_{2}=(p q)^{i}, \quad u_{4}=(q p)^{i}, i \geq 1, \quad u_{3}=p(q p)^{n}, n \geq 0
$$


where $p q$ is primitive. If $z_{\mathrm{L}}=\alpha$, then, by Lemma 2, $\alpha \beta$ is not primitive, which contradicts Corollary 2 . Hence assume $z_{\mathrm{L}}=\beta$, which implies $u_{1}=u_{3}$. Similarly $u_{5}=u_{3}$ and we have $p(q p)^{n}(p q)^{i}=u_{1} u_{2}=\alpha=$ $u_{4} u_{5}=(q p)^{i} p(q p)^{n}$. By Lemma 5, then $p$ and $q$ commute, which is again a contradiction.

\section{The Uniqueness of the Bi-infinite Word}

In [KMP2], cf. Theorem 1 it was proved that if the rank of the set $X$ equals to $\operatorname{card}(X)$, then the number of $X$-ambiguous bi-infinite words is finite. In this section we shall prove that in the two-element case, for each set $X$, there is at most one $X$-ambiguous bi-infinite word. This holds also in the case when $r_{\mathrm{c}}(X)=1$, since then both elements of $X$ are powers of a common word $t$ and the only possible bi-infinite word is $t^{\mathbb{Z}}$. The situation is also trivial in the case when roots of elements of $X=\{\alpha, \beta\}$ are conjugates: by Corollary 3 the only possible bi-infinite word is $w=\alpha \mathbb{Z}=\beta^{\mathbb{Z}}$. So we need to consider only the case when the roots of $\alpha$ and $\beta$ are not conjugates.

In this case, by Theorem 2, we know that an $X$-ambiguous bi-infinite word must be of the form $\left(\alpha \beta^{n}\right)^{\mathbb{Z}}$ or $\left(\alpha^{n} \beta\right)^{\mathbb{Z}}$. Moreover, since $w$ has two $X$-factorizations, the word $\alpha \beta^{n}$ or the word $\alpha^{n} \beta$ cannot be primitive, by Lemma 2 .

As we stated in the previous section, if $\alpha$ and $\beta$ are conjugates, then the words $\alpha \beta^{n}$ and $\alpha^{n} \beta$ are primitive for all $n$. Now, we shall show a similar result for $\alpha, \beta$ being non-conjugates, i.e., we shall show that at most one word in the set of words $\left\{\alpha \beta^{n} ; n \geq 1\right\} \cup\left\{\alpha^{m} \beta ; m \geq 1\right\}$ is not primitive. By this result, we have that also in the last case there is at most one $X$-ambiguous bi-infinite word. We need two lemmas.

Lemma 8. Let $\alpha, \beta$ be primitive and not conjugates. Then for any $n, m \geq 0$ with $n \neq m$, at most one of the words $\alpha \beta^{n}$ and $\alpha \beta^{m}$ is not primitive.

Proof. Assume the contrary that both $\alpha \beta^{n}, \alpha \beta^{m}$ are non-primitive with $m<n$. For $m=0$ the claim is obvious, so we can assume $m \geq 1$, and so $n \geq 2$. We can write

$$
\left.\begin{array}{c}
\alpha \beta^{n}=s^{i} \\
\alpha \beta^{m}=t^{j}
\end{array}\right\} \quad \text { and therefore also } s^{i}=t^{j} \beta^{n-m},
$$

where $s, t$ are primitive and $i, j \geq 2$. Now if $n-m \geq 2$, then, by Lemma $\emptyset, s, t$ and $\beta$ are powers of a common word, and so are $\alpha$ and $\beta$, which is a contradiction. So we can assume $m=n-1$, and thus equation (II) simplifies to $s^{i}=t^{j} \beta$.

Now if $|s| \leq(n-1)|\beta|$, then $|\beta|+|s| \leq\left|\beta^{n}\right|$, so that, by the equation $\alpha \beta^{n}=s^{i}$, words $\mathbb{N}_{\beta}$ and $\mathbb{N}_{s}$ have a common suffix of a length at least $|\beta|+|s|$. Applying Lemma 1 we conclude that words $s$ and $\beta$ are powers of a common word, which again yields to a contradiction. So we have

$$
\begin{aligned}
& |s|>(n-1)|\beta| \geq|\beta|, \text { and similarly, } \\
& |t|>(m-1)|\beta|=(n-2)|\beta| .
\end{aligned}
$$

Inequality (22) together with equation (II) implies $\left|t^{j}\right|=i|s|-|\beta|>(i-1)|s|$. So, if $i \geq 3$ we have $\left|t^{j}\right|>2|s|$, and since $j \geq 2$ also that $\left|t^{j}\right|>|s|+|t|$. Then equation (11) and Lemma 1 implies that $s$ and $t$ commutes which leads to a contradiction. Hence we can assume $i=2$. 
If $|t|+|\beta| \leq|s|$, then using equation (II) we derive inequality $\left|t^{j}\right| \geq(i-1)|s|+|t|$ and, by Lemma 1, we have a contradiction again. Hence we may assume that

$$
|t|+|\beta|>|s| .
$$

Now consider the case $n \geq 3$. We have

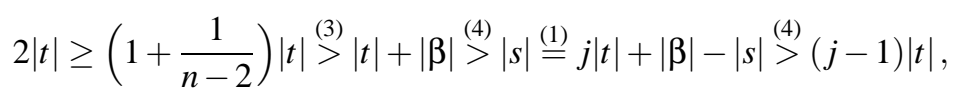

which implies that $j=2$ and also that $|t|>|\beta|$ by the two first inequalities. The second inequality and the equation $\alpha \beta^{n}=t^{2}$ imply that $t=x \beta$ for some $x \neq 1$. Thus equation (11) yields to $s^{2}=t^{2} \beta=x \beta x \beta \beta$, which implies that $|\beta|$ is an even integer, $|x \beta|<|s|$ and $\frac{3}{2}|\beta|<|s|$. Hence, we can write $s=x \beta y=z \beta_{2} \beta$ for some $y, z \neq 1$, where $|y|=\left|\beta_{2}\right|=\frac{|\beta|}{2}, \beta=\beta_{1} \beta_{2}$ and $|x|=|z|$. We can divide this equation into two parts: $x=z$ and $\beta y=\beta_{2} \beta$, where the second one, by Lemma 2, contradicts the primitiveness of $\beta$.

The last case we have to analyze is $n=2$. Now if $|t| \geq|\beta|$, then, by (5), we have $2|t| \geq|t|+|\beta|>$ $(j-1)|t|$ and $j=2$, which is again the previous case. So consider the case $|t|<|\beta|<|s|$, where the second inequality comes from (22). By the equations $\alpha \beta=t^{j}$ and $\alpha \beta^{2}=s^{2}$ we can write $\beta=x t$ and $s=y t$ for some $x, y \neq 1$. Hence equation (II) leads to $y t y t=t^{j} \beta$. We have $|y t|=|s|=\left|t^{j}\right|+|\beta|-|s|<\left|t^{j}\right|$, so that we can write $y t z=t^{j}, z \neq 1$. Now either $t$ is not primitive by Lemma 2, or $t$ matches with some $t$ in $t^{j}$, but then we have $y=t^{k}$, and hence also $s=t^{k+1}$, so that words $t, s$ are powers of a common word. In both cases we arrive to a contradiction.

Lemma 9. Let $\alpha, \beta$ be primitive and not conjugates. Then for any $n, m \geq 0$ with $(n, m) \neq(1,1)$, at most one of the words $\alpha \beta^{n}$ and $\alpha^{m} \beta$ is not primitive.

Proof. Cases $m=0$ and $n=0$ are trivial. The case $m=1$ is a special case of Lemma 8. In the case $n=1$ we can exchange $\alpha$ and $\beta$ and take reverses of words, and we are again in the case $m=1$. We shall use this reasoning again later, so let us call it the reverse argument. Consider $n, m \geq 2$ and assume the contrary that $\alpha \beta^{n}=s^{i}, \alpha^{m} \beta=t^{j}$, where $i, j \geq 2$ and $s, t$ are primitive. Using the same argument as in the proof of the previous lemma we have

$$
|s|>(n-1)|\beta| \geq|\beta|, \quad|t|>(m-1)|\alpha| \geq|\alpha| .
$$

Hence

$$
\begin{gathered}
|\alpha|=i|s|-n|\beta|>(i n-i-n)|\beta|, \\
|\beta|=j|t|-m|\alpha|>(j m-j-m)|\alpha|,
\end{gathered}
$$

which implies that

$$
[(i-1)(n-1)-1] \cdot[(j-1)(m-1)-1]<1 .
$$

So we have either $i=n=2$, or $j=m=2$. Now by the reverse argument the first case is equivalent to the second one, so it is enough to consider only the case $j=m=2$. If $|t|<|\beta|$, then, by (6), we obtain

$$
|\alpha| \stackrel{(\text { (6) }}{<}|t|<|\beta| \stackrel{(\text { (6) }}{<}|s| \text {. }
$$

Together with (7) we have $(i-1)(n-1)-1<1$, which implies that also $i=n=2$. Now again we can apply the reverse argument and the inequality $|s|>|\alpha|$ transforms to the inequality $|t|>|\beta|$. So, without 
loss of generality, we can assume that $|t|>|\beta|$. We have the situation depicted in Figure 13, where $\beta=u_{1} u_{2}$ with $\left|u_{1}\right|=\left|u_{2}\right|=\frac{1}{2}|\beta|$ and $\alpha=\alpha^{\prime} u_{1}=u_{2} \alpha^{\prime}$.

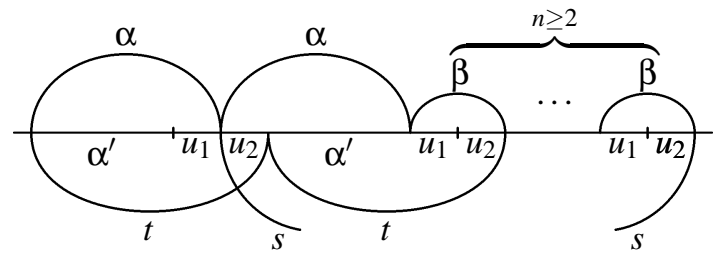

Fig. 13. The situation when $\alpha^{2} \beta=t^{2}$ and $\alpha \beta^{n}=s^{i}$ with $n \geq 2$.

Since $u_{2} \alpha^{\prime}=\alpha^{\prime} u_{1}$, Lemma 3 gives us

$$
\left.\begin{array}{rl}
u_{2} & =(p q)^{k} \\
u_{1} & =(q p)^{k} \\
\alpha^{\prime} & =p(q p)^{l}
\end{array}\right\} \quad \text { and therefore } \quad\left\{\begin{array}{l}
\alpha=p(q p)^{k+l} \\
\beta=(q p)^{k}(p q)^{k}
\end{array}\right.
$$

where $k \geq 1, l \geq 0$ and $p q$ is primitive. We may assume $p, q \neq 1$. Now considering the last occurrence of $s$ in Figure 13 we can, by (6), write $s=s^{\prime} \beta=s^{\prime}(q p)^{k}(p q)^{k}$. We also have

$$
|s|=|\alpha|+n|\beta|-(i-1)|s| \leq|\alpha|+n|\beta|-|s| \stackrel{(6)}{<}|\alpha|+|\beta|,
$$

which yields

$$
s^{\prime}(q p)^{k}(p q)^{k} r=s r=\alpha \beta=\underbrace{p(q p)^{2 k+l}}_{w}(p q)^{k},
$$

for some $r \neq 1$. The first occurrence of $q p$ in $s$ after $s^{\prime}$ must match with $q p$ in $w$, otherwise $q p$ is not primitive. But then, since $r \neq 1$, the first occurrence of $p q$ in $s$ after $s^{\prime}(q p)^{k}$ matches with some $q p$ in $w$, so we have $p q=q p$, which is again a contradiction with the primitiveness of $p q$.

As a consequence of Lemmas $\square$ and 9 we have the following corollary:

Corollary 4. Let $\alpha, \beta$ be primitive and not conjugates. Then at most one word in the set $\left\{\alpha \beta^{n} ; n \geq\right.$ $1\} \cup\left\{\alpha^{m} \beta ; m \geq 1\right\}$ is not primitive.

Finally, we can state the result of this section, which is a consequence of Corollary 4 and the considerations in the beginning of this section.

Theorem 3. Consider set $X=\{\alpha, \beta\}$ with $\alpha, \beta \in \Sigma^{+}$. There is at most one $X$-ambiguous bi-infinite word over $\Sigma$.

\section{The Existence of the Bi-infinite Word}

We consider again only the two-element case in this section. In the previous section we proved that there is at most one $X$-ambiguous bi-infinite word. It is natural to ask when such a word exists. It is easy to see that there are sets $X$ for which there is no $X$-ambiguous bi-infinite word. For example, take a set 
$X=\Sigma=\{a, b\}$. We say that a family of sets of words with the same cardinality $t$ is parameterizable if it can be described in terms of $t$ formulas with word and integer parameters. We shall prove now that the family of binary sets $X$ for which there exists an $X$-ambiguous bi-infinite word is parameterizable.

In case $(i)$ of Theorem 2, when words of $X$ are powers of a common word $t$, the bi-infinite word $t^{\mathbb{Z}}$ has infinitely many $X$-factorizations. In particular, in the case there is always an $X$-ambiguous bi-infinite word. In case (ii), when roots of words in $X=\{\alpha, \beta\}$ are conjugates, the bi-infinite word $\alpha^{\mathbb{Z}}=\beta^{\mathbb{Z}}$ has exactly two different $X$-factorizations, so it is $X$-ambiguous.

Consider now the last case (iii), and the set $X=\{\alpha, \beta\}$. By Theorem 2 , an $X$-ambiguous bi-infinite word is of the form $\left(\alpha \beta^{n}\right)^{\mathbb{Z}}$, where $\alpha \beta^{n}$ is not primitive, or $\left(\alpha^{n} \beta\right)^{\mathbb{Z}}$, where $\alpha^{n} \beta$ is not primitive, i.e., there are $n \geq 1, i \geq 2$ and $s \in \Sigma^{+}$such that

$$
\alpha \beta^{n}=s^{i} \quad \text { or } \quad \alpha^{n} \beta=s^{i} .
$$

Conversely, if for some $n \geq 1$ and $i \geq 2$ at least one of equations (8) has a solution, then clearly the bi-infinite word $\left(\alpha \beta^{n}\right)^{\mathbb{Z}}$ (resp. $\left(\alpha^{n} \beta\right)^{\mathbb{Z}}$ ) has exactly $i$ shiftequivalent, but different $X$-factorizations. We formalize this as a lemma.

Lemma 10. Let $X=\{\alpha, \beta\} \subseteq \Sigma^{+}$be a set of two non-commuting words such that their roots are not conjugates. Then there is an $X$-ambiguous bi-infinite word if and only if one of the equations $\alpha \beta^{n}=s^{i}$ and $\alpha^{n} \beta=s^{i}$, with $n \geq 1, i \geq 2$, has a solution.

We shall also give a characterization of the solutions of the equations (8). We need the following lemma.

Lemma 11. The all nonperiodic solutions of the equation

$$
u_{1} u_{2}=u_{3}\left(u_{2} u_{3}\right)^{m}, \quad m \geq 1
$$

are of the form

$$
\begin{aligned}
& u_{3}=q p \\
& u_{2}=p(q p)^{k} \\
& u_{1}=u_{3}\left(u_{2} u_{3}\right)^{m-1} p q,
\end{aligned}
$$

where $p, q \in \Sigma^{+}, k \geq 0$.

Proof. It is easy to check that (10) is really a solution of equation (97). Now we shall prove that if equation (9) has a nonperiodic solution, then it is of the form (10). We proceed by induction.

Consider first the case $m=1$. We have the equation $u_{1} u_{2}=u_{3} u_{2} u_{3}$. It is obvious that $\left|u_{1}\right|>\left|u_{3}\right|$, so we can write $u_{1}=u_{3} t$. The equation transforms into $t u_{2}=u_{2} u_{3}$, which has, by Lemma B, the only solutions $t=p q, u_{3}=q p$ and $u_{2}=p(q p)^{k}, k \geq 0$. This implies that $u_{1}=q p p q$, so we have a solution of the form (II)) for $m=1$.

Consider now equation (99) with $m \geq 2$. Again we have $\left|u_{1}\right|>\left|u_{3}\right|$, so we can substitute $u_{1}=u_{3} t$ and equation (9) becomes $t u_{2}=u_{2} u_{3}\left(u_{2} u_{3}\right)^{m-1}$. By Lemma 3, we have $t=u v, u_{3}\left(u_{2} u_{3}\right)^{m-1}=v u, u_{2}=$ $u(v u)^{l}$. If $l \geq 1$, then $|v u|=\left|u_{3}\left(u_{2} u_{3}\right)^{m-1}\right| \geq 2|u|+|v|+\left|u_{3}\right|$. This implies that $u=u_{3}=1$, which leads to a periodic solution. Hence, consider the case $l=0$. We have $u_{2}=u, u_{1}=u_{3} u_{2} v$ and $v u_{2}=$ $u_{3}\left(u_{2} u_{3}\right)^{m-1}$. Now we can apply induction hypothesis on the last equation and we obtain that all noncommuting solutions are of the form

$$
u_{3}=q p, \quad u_{2}=p(q p)^{k}, \quad v=u_{3}\left(u_{2} u_{3}\right)^{m-2} p q, \quad k \geq 0,
$$


which implies $u_{1}=u_{3} u_{2} v=u_{3}\left(u_{2} u_{3}\right)^{m-1} p q$. We obtained exactly solution (10), which completes the proof.

The following lemma gives us the characterization of solutions of equation ( 8 (8) and hence also of sets $X$ allowing an $X$-ambiguous bi-infinite word in case (iii).

Lemma 12. Assume that $\alpha$ and $\beta$ do not commute. All solutions of the equation $\alpha \beta^{n}=s^{i}$ satisfying $n \geq 1$, $i \geq 2$ are

$$
\begin{aligned}
\beta & =p(q p)^{j}, \\
s & =q p \beta^{n-1}, \\
\alpha & =s^{i-1} \beta^{-1} p q,
\end{aligned}
$$

where $p, q \in \Sigma^{+}, j \geq 0$ and $j<i$ if $n=1$.

Proof. It is easy to check that (II) is a solution of equation (8). For the converse implication we analyze 3 cases.

Case 1. Assume that $|s|>\left|\beta^{n}\right|$. Then we have $\alpha=s^{i-1} q$ and $s=q \beta^{n}$ for some $q \neq 1$. This is solution (II) for $j=0, p=\beta$.

Case 2. Assume that $|s|<\left|\beta^{n}\right|$ and $n=1$. The situation is depicted in Figure 14.

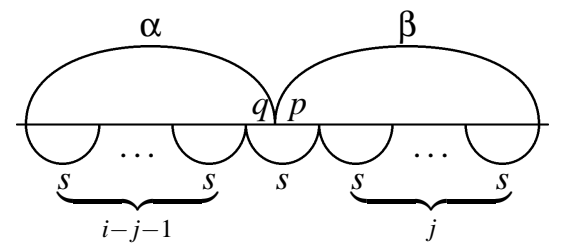

Fig. 14. The situation when $|s|<\left|\beta^{n}\right|$ and $n=1$.

Directly from the figure we can write

$$
\beta=p(q p)^{j}, \quad s=q p, \quad \alpha=q(p q)^{i-j-1},
$$

where $p, q \neq 1$ and $j<i$. Since

$$
s^{i-1} \beta^{-1} p q=(q p)^{i-1}\left[p(q p)^{j}\right]^{-1} p q=(q p)^{i-j-1} q=\alpha,
$$

we have solution (III) for $n=1$.

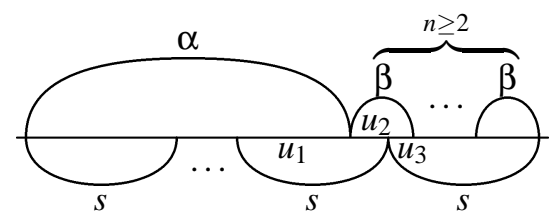

Fig. 15. The situation when $|s|<\left|\beta^{n}\right|$ and $n \geq 2$. 
Case 3. Finally assume that $|s|<\left|\beta^{n}\right|$ and $n \geq 2$. Since we are looking for non-commuting solutions, necessarily $|s|>\left|\beta^{n-1}\right|$ (see the proof of Lemma 8). Hence, we have a situation shown in Figure 15 . According to this figure we can write $\beta=u_{2} u_{3}, \alpha=s^{i-2} u_{1}$ and $u_{1} u_{2}=s=u_{3} \beta^{n-1}=u_{3}\left(u_{2} u_{3}\right)^{n-1}$, which is equation (9). Now, Lemma 11 implies

$$
\begin{aligned}
\beta & =u_{2} u_{3}=p(q p)^{k+1}=p(q p)^{j}, \text { for } j=k+1, \\
s & =u_{1} u_{2}=u_{3}\left(u_{2} u_{3}\right)^{n-2} p q p(q p)^{k}=q p \beta^{n-2} \beta=q p \beta^{n-1}, \text { and } \\
\alpha & =s^{i-2} u_{1}=s^{i-2} u_{3}\left(u_{2} u_{3}\right)^{n-2} p q=s^{i-2} q p \beta^{n-2} \beta \beta^{-1} p q=s^{i-1} \beta^{-1} p q .
\end{aligned}
$$

This is exactly solution (11).

The following theorem summarizes the previous results.

Theorem 4. Consider set $X \subseteq \Sigma^{+}$with $\operatorname{card}(X)=2$. There exists an $X$-ambiguous bi-infinite word if and only if one of the following conditions is satisfied:

(i) $X=\left\{p^{n}, p^{m}\right\}$, where $p \in \Sigma^{+}$and $n, m \geq 1$,

(ii) $X=\left\{(p q)^{n},(q p)^{m}\right\}$, where $p, q \in \Sigma^{+}$and $n, m \geq 1$,

(iii) $X=\{\alpha, \beta\}$, where

$$
\beta=p(q p)^{j}, \quad \alpha=\left(q p \beta^{n-1}\right)^{i-1} \beta^{-1} p q,
$$

for $p, q \in \Sigma^{+}, n \geq 1, i \geq 2, j \geq 0$ and if $n=1$, then $j<i$.

Notice, that in the last case of Theorem $\emptyset$ the occurrence of $\beta^{-1}$ can be eliminated, but we prefer this form for its simplicity. This theorem shows that the family of the two-element sets $X$, such that there exists an $X$-ambiguous bi-infinite word, is parameterizable. Such a characterization does not help us, if we want to decide whether there is an $X$-ambiguous bi-infinite word for the certain set $X$, but we can use it to generate all such sets.

Example 3. Let us choose in (III) $p=a, q=b, n=2, i=2$ and $j=2$. We have

$$
\beta=a b a b a, \quad s=b a a b a b a, \quad \alpha=b a a b .
$$

The bi-infinite word $\left(\alpha \beta^{2}\right)^{\mathbb{Z}}$ has two different $X$-factorizations:

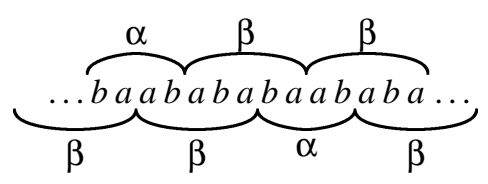

\section{Conclusions and Open Problems}

Our Theorem $凤$ is closely related to the main result of [IRIR], where it is characterized when a finite word can have two disjoint $X$-interpretations for a binary set $X$. Our result could be concluded, with some effort, from the considerations in this paper. However, our proof is simpler, due to the use of the graph lemma (Lemma 5), and moreover directly formulated to obtain a defect type of theorems.

We pose an open problem asking whether Theorem 2 can be extended to arbitrary sets. 
Open problem 1. Let $X \subseteq \Sigma^{+}$be a finite set such that $r_{\mathrm{c}}(X)=\operatorname{card}(X)$. Does there exist a bi-infinite word $w$ having two $X$-factorizations $F_{1}$ and $F_{2}$ satisfying:

(i) both $F_{1}$ and $F_{2}$ contain all elements of $X$, and

(ii) $F_{1}$ and $F_{2}$ are not shiftequivalent?

Observe here that in the case of a two-element set $X$ the answer to this problem is negative, but without the assumption that all elements of $X$ occur in both factorizations the answer is trivially positive.

The answer is also positive if the condition $r_{c}(X)=\operatorname{card}(X)$ is replaced by a weaker one involving the free rank: $r_{\mathrm{f}}(X)=\operatorname{card}(X)$. This is verified by Example 11.

Another open problem asks whether Corollary 3 can be generalized for an arbitrary finite $X$.

Open problem 2. Let $X \subseteq \Sigma^{+}$be a finite set satisfying $r_{\mathrm{c}}(X)=\operatorname{card}(X)$. Suppose that primitive roots of all elements of $X$ are conjugates and that a bi-infinite word $w$ has at least two different $X$-factorizations. Are all $X$-factorizations of $w$ of the form $\alpha^{\mathbb{Z}}$, where $\alpha \in X$ ?

Example 4. The answer to the above question is negative if we omit the assumption $r_{\mathrm{c}}(X)=\operatorname{card}(X)$. Indeed, let $X=\left\{\alpha_{1}, \alpha_{2}, \alpha_{3}\right\}$, where $\alpha_{1}=b a a, \alpha_{2}=a b a, \alpha_{3}=a a b$. Then clearly $\alpha_{1} \sim \alpha_{2} \sim \alpha_{3}$ and the word $($ abaaa $))^{\mathbb{Z}}$ has two different, and even non-shiftequivalent, $X$-factorizations: $\left(\alpha_{1} \alpha_{2}\right)^{\mathbb{Z}}$ and $\left(\alpha_{2} \alpha_{3}\right)^{\mathbb{Z}}$.

\section{Acknowledgment}

The author is grateful to Professor Juhani Karhumäki and to Wojciech Plandowski for useful discussions.

\section{References}

[Br] Bruyère, V., Codes, Chapter 7, in: M. Lothaire (ed), Algebraic combinatorics on words (to appear).

[CK] Choffrut, C., Karhumäki, J., Combinatorics of words, in: G. Rozenberg and A. Salomaa (eds), Handbook of Formal Languages, Vol. I, Springer, 329-438, 1997.

[HK] Harju, T., Karhumäki, J., On the defect theorem and simplifiability, Semigroup Forum 33, 199 $217,1986$.

[KMP1] Karhumäki, J., Maňuch, J., Plandowski, W., On defect effect of bi-infinite words, in: MFCS'98, Lecture Notes in Computer Science 1450, 674-682, 1998.

[KMP2] Karhumäki, J., Maňuch, J., Plandowski, W., A defect theorem for bi-infinite words, (to appear in a special issue of TCS).

[Lo] Lothaire, M., Combinatorics on words, Addison-Wesley, Encyclopedia of Mathematics and its Applications 17, 1983.

[IRIR] Le Rest, E., Le Rest, M. Sur la combinatoire des codes a deux mots, Theoretical Computer Science 41, 61-80, 1985. 
[LeS] Lentin, A., Schützenberger, M.P., A combinatorial problem in the theory of free monoids, in: R.C. Bose and T.W. Dowling (eds), Combinatorial Mathematics and its Applications, Univ. North Carolina Press, 128-144, 1969.

[LyS] Lyndon, R.C., Schützenberger, M. P., The equation $a^{m}=b^{n} c^{p}$ in a free group, Michigan Mathematical Journal 9, 289-298, 1962. 